\title{
The Antimicrobial Peptide, Nisin, Synergistically Enhances the Cytotoxic and Apoptotic Effects of Rituximab Treatment on Human Burkitt's Lymphoma Cell Lines
}

\author{
Pantea Mohammadi ${ }^{1}$, Mina Zangeneh ${ }^{1}$, Hamid-Reza Mohammadi-Motlagh ${ }^{1}$, \\ Fatemeh Khademi*1
}

\begin{abstract}
Background: Non-Hodgkin's lymphomas comprise the most common hematological cancers worldwide and consist of a heterogenous group of malignancies affecting the lymphoid system. Treatment of non-Hodgkin's lymphoma has been significantly enhanced with the addition of Rituximab to the standard chemotherapy regimen. However, even with the advancement of treatment patients continue to relapse and develop resistance to Rituximab, rendering subsequent treatments unsuccessful. The use of novel drugs with unique antitumor mechanisms has gained considerable attention. In this study, we explored the in vitro anti-cancer effects of the combined therapy of Rituximab and Nisin on human Burkitt's lymphoma cells.

Methods: The human Burkitt's lymphoma cells lines, Raji and Daudi, were treated with Nisin, Rituximab, or a combination of the two agents at various concentrations. Cytotoxicity following treatment was determined using cell viability assay. The degree of apoptosis was verified via flow cytometric analysis using FITC annexin V/PI staining.

Results: Our findings show that the combined treatment of Rituximab and Nisin results in a more significant reduction in the survival of Raji and Daudi Burkitt's lymphoma cells, compared to Nisin or Rituximab treatment alone. Additionally, our results indicate that Nisin can induce a significant degree of apoptosis in the Burkitt's lymphoma cells compared to the negative controls. However, the addition of Nisin to the Rituximab treatment synergistically enhances the apoptotic antitumor effect.

Conclusions: This study demonstrates the synergistic antitumor effect of Nisin treatment in vitro to enhance tumor cell apoptosis and the potential value of Nisin as an adjunct therapy in the treatment of lymphoma.
\end{abstract}

Keywords: Apoptosis, Burkitt's lymphoma, Cell viability, Combination, Nisin, Rituximab.

\section{Introduction}

Non-Hodgkin's lymphomas (NHLs) are one of the most common malignancies worldwide and include a heterogenous group of diseases that arise from malignant lymphocytes. One of the many subtypes include Burkitt's lymphoma, which is a highly aggressive form of B cell NHL (1). Although patients with NHLs have a high initial response rate to chemotherapy, it is common for patients to ultimately relapse and develop drug resistance. Thus, the subsequent chemotherapy regimen is unable to successfully eliminate the tumor cells (2). The use of antibodymediated immunotherapy, alone or in combination with chemotherapy, has been shown to improve the overall survival rate. Anti-CD20 antibodies are a promising candidate in the treatment of NHLs. Rituximab is the first chimeric anti-CD20 monoclonal antibody (mAb) used to treat B cell disorders, including NHLs such as chronic lymphocytic leukemia. The use of Rituximab has also been demonstrated in the 
treatment of autoimmune diseases, such as multiple sclerosis $(3,4)$. Rituximab acts via binding to $\mathrm{CD} 20$ which induces antibodydependent cell-mediated cytotoxicity (ADCC), complement dependent cytotoxicity (CDC), and to a lesser extent, apoptosis of malignant cells (57). Rituximab is frequently used alone or in combination with chemotherapy to treat lymphoma, however patients often develop a resistance to Rituximab and the cancer may relapse. These findings highlight the importance of enhancing existing treatments to improve the clinical outcome for these patients.

A growing number of studies have revealed the antitumor properties of antimicrobial peptides which has led to considerable interest in their use as an alternative cancer treatment (8-14). These small molecules have potent tissue penetration and uptake by cancer cells. They exert their cytotoxic effects through both their intrinsic activity or synergistically interacting with existing therapeutics, enhancing their anticancer properties (15). Nisin is a type of antimicrobial peptide that is 34 amino acid residues in size and produced the Lactococcus lactis bacterium (16). Bacteriocins, including Nisin, have become a promising candidate showing great potential in combating various infectious diseases $(15,17)$. Nisin was approved by the Food and Drug Administration (FDA) in 1988 as a food preservative safe for human use. The World Health Organization (WHO) has also confirmed its safety and it has been shown to have no cytotoxic effects on the human body. While antimicrobial peptides have a long history of use in the food industry to prevent the growth of bacteria, they have recently gained considerable attention for their antitumor activity. Several studies have shown the antitumor properties of Nisin, including its ability to induce apoptosis in gastrointestinal, hepatic, and K562 cancer cell lines (8), inhibit head and neck squamous cell carcinoma (HNSCC) tumorigenesis (11), enhance cell death and apoptosis in colon cancer cell lines (10), and decrease cell proliferation and induce apoptosis in melanoma cells (9). Nisin exerts its effect by creating pores in the cell membrane, altering the electrical potential of the cell (18). The exact role of calcium in Nisin dependent cell death has yet to be fully elucidated. However, since the pores created by Nisin lead to a net influx of calcium ions, calcium may hold a critical role in the induction of apoptosis and inhibition of cell proliferation (17). In this study, we investigated the cytotoxic effect of Nisin in human Burkitt's lymphoma cell lines and explored the potential synergistic effect of Nisin on Rituximab-induced apoptosis and cytotoxicity in vitro.

\section{Materials and methods Cell culture}

Human Raji and Daudi Burkitt's lymphoma cells were prepared from the National Cell Bank of Iran (Tehran, Iran). Antibiotic-free RPMI 1640 medium with $10 \%$ fetal bovine serum (FBS) (Gibco, Grand Island, NY) was used for maintaining the cells at $37{ }^{\circ} \mathrm{C}$ in a humidified incubator with $5 \% \mathrm{CO}_{2}$.

\section{Cell viability assay}

To determine the effect of Nisin Z (Handary, Belgium) on Burkitt's lymphoma cell viability alone or in combination with Rituximab treatment, the 3-(4,5-dimethylthiazol-2-yl)-2,5diphenyl tetrazolium bromide (MTT, SigmaAldrich) colorimetric assay was used. Briefly, $5 \times 10^{4}$ Daudi cells, Raji cells, or peripheral blood mononuclear cells (PBMCs) were plated in a 96well cell culture plate. Following plating, $10 \mu \mathrm{l}$ of Nisin or Rituximab was added to each well containing $90 \mu \mathrm{l}$ RPMI 1640. The effect of treatment was tested as various concentrations including 4, 40, 400, and $4000 \mu \mathrm{g} / \mathrm{mL}$ for Nisin, and $0.1,1,10$, and $100 \mu \mathrm{g} / \mathrm{mL}$ for Rituximab in a final mixture of $100 \mu \mathrm{l} /$ well. The concentrations used for the combined Nisin and Rituximab treatment were the IC50 concentration for each agent, which is the concentration needed to reduce cell viability by $50 \%$. For the negative controls, Daudi and Raji cells were treated with phosphate buffer saline (PBS). Next, MTT $(0.5 \mathrm{mg} / \mathrm{mL})$ was added 72 hours after treatment, and incubated for 3 hours at $37{ }^{\circ} \mathrm{C}$. After centrifugation at $2000 \mathrm{~g}$ for 5 minutes and discarding the supernatant $100 \mu \mathrm{l}$ dimethyl sulfoxide (DMSO) was added. The optical densities of each well were recorded using a 96well microplate reader (Bio-Rad Laboratories 
Inc. Philadelphia, PA) at $570 \mathrm{~nm}$. The following equation was used to estimate the percentage of cell viability: Cell viability $(\%)=\left[\mathrm{A}_{570}\right.$ (sample) $/$ A570 (control)] $\times 100$. All experiments were performed at least three times.

\section{Apoptosis assay}

The effect of Nisin $\mathrm{Z}$ alone or in combination with Rituximab on Burkitt's lymphoma cell apoptosis was determined using a direct staining method and a flow cytometry assay using FITC annexin $\mathrm{V}$ apoptosis detection kit with PI (BioLegend, San Diego, CA). In brief, cells were plated in 12-well plates at $1 \times 10^{6}$ cells $/ \mathrm{mL}$. Subsequently, $100 \mu \mathrm{l}$ of Nisin or Rituximab was added to each well containing $900 \mu \mathrm{l}$ of RPMI 1640 medium, and the plates were incubated at 37 ${ }^{\circ} \mathrm{C}$ for $48 \mathrm{~h}$. The concentration of Nisin and Rituximab were 4000 and $100 \mu \mathrm{g} / \mathrm{mL}$ in $1 \mathrm{~mL}$ mixture, respectively. These concentrations correspond to the IC50 of each agent. The effect of Nisin in combination with Rituximab was determined by adding $100 \mu \mathrm{l}$ of Nisin at a concentration of $4000 \mu \mathrm{g} / \mathrm{mL}$ and $100 \mu \mathrm{l}$ of Rituximab at a concentration of $100 \mu \mathrm{g} / \mathrm{mL}$. The negative controls were incubated with PBS. For the direct staining method, Annexin V/PI staining was used according to the manufacturer's instructions. The stained cells were visualized under a Nikon fluorescence microscope. The amount of apoptosis was assayed using flow cytometry analysis of cells stained with Annexin V/PI. Stained cells were analyzed using the Attune NxT Acoustic Focusing Cytometer (Thermo Fisher Scientific). FlowJo software version 7.6.1 was used for analysis of the results.

\section{Statistical analysis}

Data were analyzed by one-way ANOVA using SPSS software 19.0 (SPSS, Inc., Chicago, IL). Results were considered statistically significant if $\mathrm{p}$-value $<0.05$.

\section{Results}

\section{The combined treatment of Nisin and Rituximab reduces cell viability}

The cytotoxicity of Nisin, Rituximab, and the combined treatment of both agents on the Burkitt's lymphoma cell lines was investigated by MTT cell viability assay. The effect of Nisin and Rituximab on cell viability were observed to be dose dependent, as the viability decreased progressively with increasing concentrations of Nisin and Rituximab. The maximum decrease in percentage cell viability was observed following treatment with Nisin at a concentration of $4000 \mu \mathrm{g} / \mathrm{mL}$ and Rituximab at a concentration of $100 \mu \mathrm{g} / \mathrm{mL}$ in both Raji and Duadi cell lines (Figs. 1A and 1B). The IC50 of Nisin and Rituximab was estimated to be 4000 $\mu \mathrm{g} / \mathrm{mL}$ and $10 \mu \mathrm{g} / \mathrm{mL}$, respectively. For the combined treatment, the IC50 concentration of each agent was used. In the cells that received the combined treatment of Rituximab and Nisin, we observed a significant decrease in the percentage cell viability compared to treatment with Rituximab and Nisin alone at their respective IC50 concentrations ( $p<0.001)$. These findings indicate that the addition of Nisin to Rituximab treatment can enhance the degree at which cell viability is reduced (Fig. 1C). Treatment with Nisin or the combination of Nisin and Rituximab showed no cytotoxic effects on normal human B cells.

\section{Nisin and Rituximab combination induces significant apoptosis}

We further examined whether the effect of Nisin on Rituximab-mediated cytotoxicity was synergistic. As demonstrated in Figure 2A, flow cytometric analysis clearly showed that treatment with either Nisin or Rituximab at their IC50 concentrations induces moderate apoptosis in both Raji and Daudi cells, compared to the negative controls. In contrast, the combination of Nisin $(4000 \mu \mathrm{g} / \mathrm{mL})$ and Rituximab $(10 \mu \mathrm{g} / \mathrm{mL})$ resulted in a synergistic increase in apoptosis of the Burkitt's lymphoma cells (Fig. 2). Following combination treatment, about $>20 \%$ and $>40 \%$ of the Raji and Daudi cells underwent apoptosis, respectively. Flow cytometry analysis is shown in Figure 2 and the results of the Annexin V/PI staining assay are illustrated in Figure 3. Greater PS exposure and cell death were induced by the Nisin and Rituximab combined treatment compared to Nisin or Rituximab alone. 

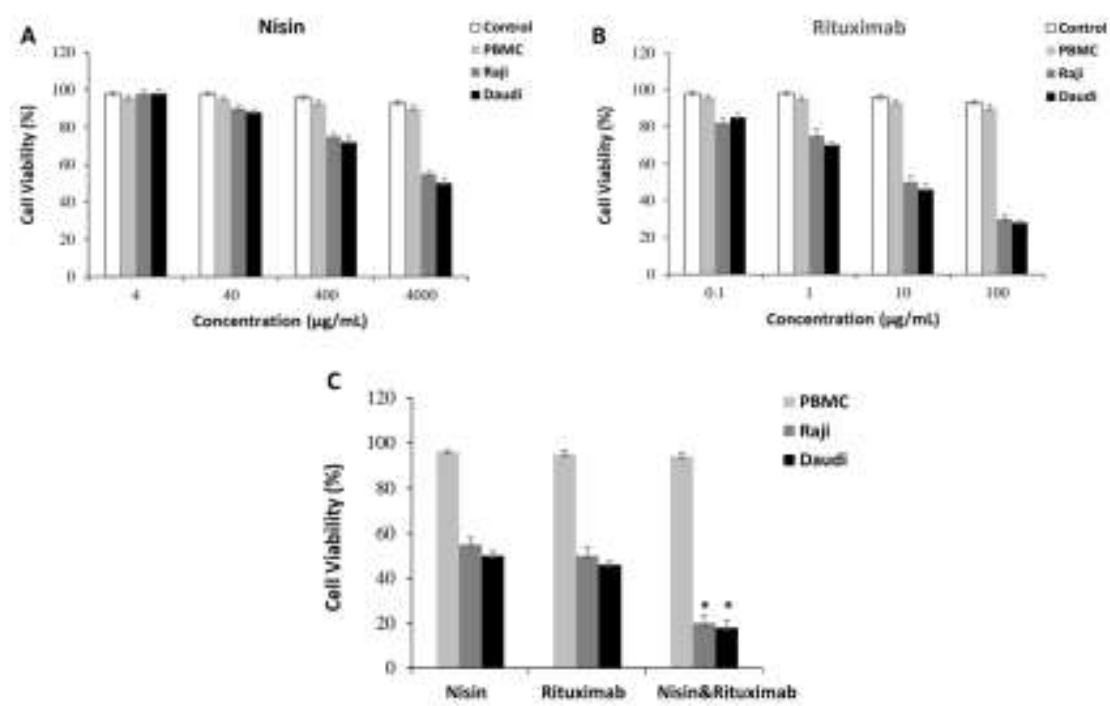

Fig. 1. Nisin significantly reduces cell viability in a dose-dependent manner and potentiates the cytotoxic effect of Rituximab. The effect of (A) Nisin, (B) Rituximab, and (C) Nisin in combination with Rituximab ( ${ }^{*} p<0.001$ compared with Nisin or Rituximab alone) on the viability of Raji and Daudi cells and PBMCs, as measured by the MTT assay. The concentration used for combination treatments with Nisin and Rituximab corresponds to the IC50 of each agent. Controls were Raji or Daudi cells incubated with PBS. Comparisons between groups were analyzed by one-way ANOVA.
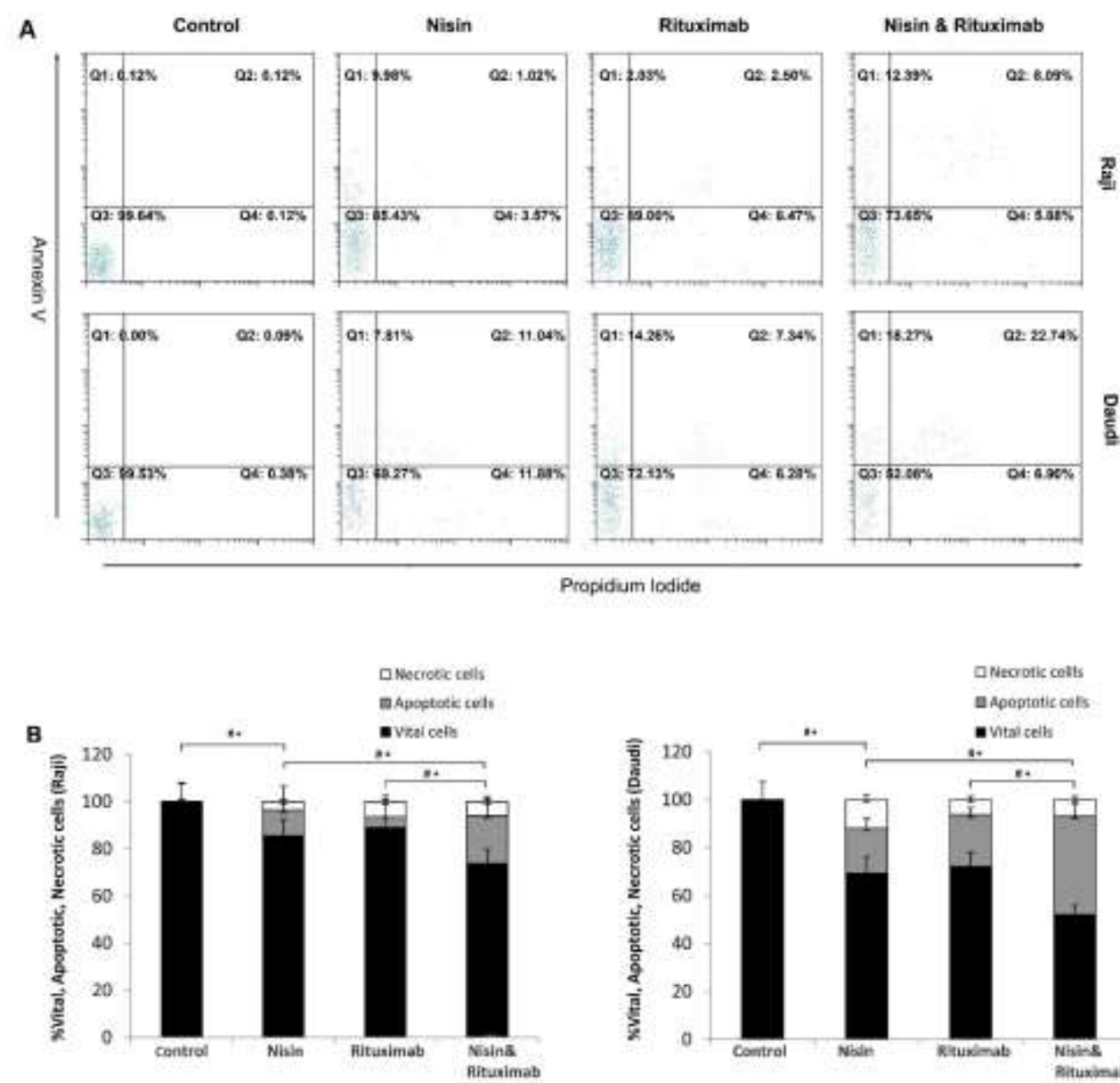

Propidium lodide

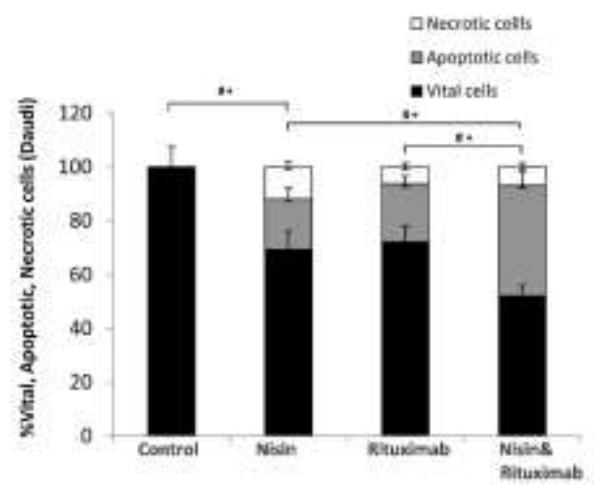

Fig. 2. Nisin induces significant cell apoptosis and potentiates the apoptotic effect of Rituximab. (A) Flow cytometry analysis was performed to determine the impact of Nisin, Rituximab, or the combined treatment on Raji and Daudi cell apoptosis. (C) Percentage of vital, apoptotic, and necrotic cells (Raji and Daudi) treated with control (PBS), Nisin (4000 $\mu \mathrm{g} / \mathrm{mL})$, Rituximab $(10 \mu \mathrm{g} / \mathrm{mL})$, and Nisin $(4000 \mu \mathrm{g} / \mathrm{mL})$ in combination with Rituximab $(10 \mu \mathrm{g} / \mathrm{mL})$. Comparisons between groups were analyzed by one-way ANOVA (\# $p<$ 0.001 vital cells; ${ }^{*} p<0.001$ apoptotic cells). 

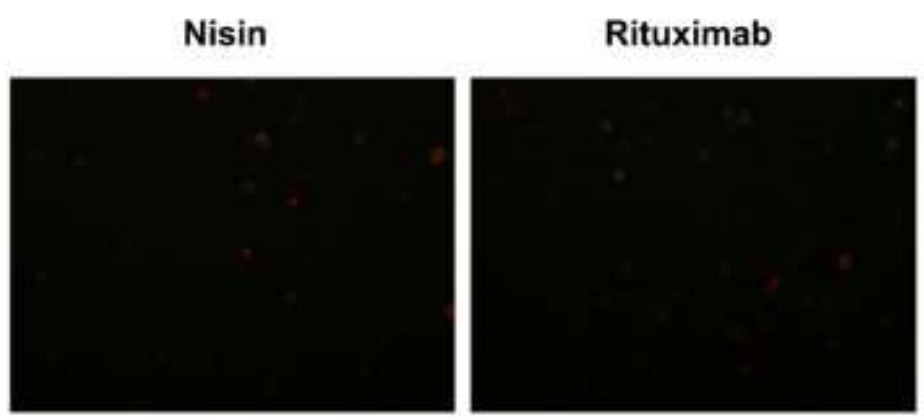

Nisin\&Rituximab

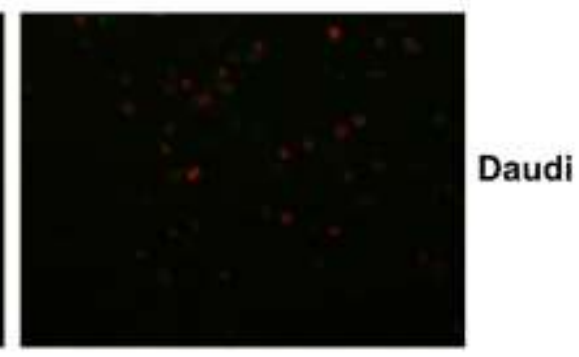

Fig. 3. Microscopic analysis of direct FITC annexin V/PI staining of Daudi cells treated with Nisin, Rituximab, and Nisin in combination with Rituximab. Greater PS exposure and cell death were induced by the combination of Nisin and Rituximab compared to Nisin or Rituximab alone.

\section{Discussion}

In this study, we provide novel evidence that Nisin can potentiate the cytotoxic effects of Rituximab and lead to a significant increase in the level of apoptosis in human Burkitt's lymphoma cell lines. The purpose of the present study was to evaluate the in vitro anti-cancer effects of the combined treatment of Nisin and Rituximab on human Burkitt's lymphoma cells. Cells received treatment with either Nisin, Rituximab, or a combination of the two agents. Our findings showed that Nisin decreases the viability of Raji and Daudi Burkitt's lymphoma cells at a concentration of $4000 \mu \mathrm{g} / \mathrm{mL}$, while the combined treatment of Nisin and Rituximab reduced cell viability significantly more than that of Nisin or Rituximab alone at their respective IC50 concentrations. These findings indicate that the addition of Nisin to Rituximab treatment may have the ability to further reduce the cell viability of Burkitt's lymphoma cell lines. To verify the level of apoptosis, flow cytometry analysis using FITC annexin V/PI staining was performed. Nisin was found to induce significantly more apoptosis compared to the negative controls. However, the combined treatment of Nisin and Rituximab was observed to synergistically increase the level of apoptosis of each Burkitt's lymphoma cell line compared to Nisin or Rituximab alone. This synergistic effect appears to be a result of the combined function of both Nisin and Rituximab in modulating apoptotic regulatory proteins. B-cell lymphoma (Bcl)-extra-large (Bcl-xL) and Bcl-2 are critical anti-apoptotic genes that promote cell survival, while Bcl-2-associated X protein (BAX) and Bcl-2 homologous antagonist/killer (BAK) are both pro-apoptotic genes that induce cell death. The expression of BAX and BAK increase the apoptotic index (bax, bak/ Bcl-xL, Bcl-2), leading to the induction of apoptosis. Nisin can induce apoptosis through increasing the apoptotic index (bax/bcl-2 ratio) both at the mRNA and protein level (10), while Rituximab selectively downregulates the expression of the anti-apoptotic Bcl$x L$ gene (7). The regulatory role of Rituximab on apoptosis, along with those exerted by Nisin, demonstrate that the combination of Nisin and Rituximab treatment may be an effective therapy for patients with treatment resistant Burkitt's lymphoma.

A growing number of studies have highlighted the antitumor properties of antimicrobial peptides (8-14). In a recent study conducted by Goudarzi et al. (8), Nisin was observed to have a cytotoxic effect on K562, AGS, HepG2, and KYSE-30 cancer cell lines. The use of Nisin-loaded nanoparticles was found to have a more cytotoxic effect than free Nisin. In a separate study, Nisin Z was shown to induce selective toxicity in the A375 melanoma cell line in comparison to nonmalignant keratinocytes. These findings suggest that the combined therapy of Nisin $\mathrm{Z}$ and antimelanoma agents may be an effective treatment option for patients with melanoma (9). Zainodini et al. (19) reported that Nisin treatment significantly increases cell death and apoptosis in the astrocytoma cell line, SW1088, compared controls. In a study conducted by Ahmadi et al. (10), treatment with Nisin was found to significantly increase the cell viability and apoptosis in the SW480 colon cancer cell line. The antiproliferative and apoptotic effects of Nisin were reported to occur at a concentration 2000-4000 $\mu \mathrm{g} / \mathrm{mL}$. This is similar to our findings in which the 
IC50 concentration of Nisin obtained in our study was about $4000 \mu \mathrm{g} / \mathrm{mL}$. Consistent with our findings, Ahmadi et al. reported that the antiproliferative effect of Nisin was dose dependent. Moreover, they reported that Nisin induces apoptosis by increasing the apoptotic index (bax/bcl-2 ratio). Furthermore, a separate study has shown that Nisin can significantly increase apoptosis by mediating calcium flux via the activation of calpain and decrease cell proliferation through cell cycle arrest in head and neck squamous cell carcinoma cells both in vitro and in vivo (11).

Although the antitumor ability of Nisin to induce apoptosis and its cytotoxic effects have been previously described, our study provides novel evidence showing that Nisin can potentiate the cytotoxic effect of Rituximab, leading to a significant increase in apoptosis in Raji and Daudi Burkitt's lymphoma cell lines.

In conclusion, our findings have shown that Nisin can reduce the viability of Raji and Daudi Burkitt's lymphoma cell lines in vitro and confirmed that Nisin reduces cell viability in a dose-dependent manner. Furthermore, the combined treatment of Nisin with Rituximab at

\section{References}

1. Singh V, Gupta D, Almasan A. Development of Novel Anti-Cd20 Monoclonal Antibodies and Modulation in $\mathrm{Cd} 20$ Levels on Cell Surface: Looking to Improve Immunotherapy Response. J Cancer Sci Ther. 2015;7(11):347-358.

2. White CA. Rituximab immunotherapy for nonHodgkin's lymphoma. Cancer Biother Radiopharm. 1999;14(4):241-50.

3. Myhr K-M, Torkildsen O, Lossius A, Bo L, Holmoy T. B cell depletion in the treatment of multiple sclerosis. Expert Opin Biol Ther. 2019;19(3):261-271.

4. Du FH, Mills EA, Mao-Draayer Y. Nextgeneration anti-CD20 monoclonal antibodies in autoimmune disease treatment. Auto Immun Highlights. 2017;8(1):12.

5. Reff ME, Carner K, Chambers KS, Chinn PC, Leonard JE, Raab R, et al. Depletion of B cells in vivo by a chimeric mouse human monoclonal antibody to CD20. Blood. 1994;83(2):435-45. their respective IC50 concentrations results in a significant reduction in cell viability compared to Nisin or Rituximab alone. These findings suggest that the addition of Nisin can enhance the ability of Rituximab treatment to reduce the viability of Burkitt's lymphoma cell lines. Furthermore, our findings show that Nisin can induce apoptosis in Burkitt's lymphoma cell lines. Similarly, the combined treatment of Nisin with Rituximab works synergistically to enhance the level of apoptosis in these lymphoma cells. Our findings demonstrate the importance of Nisin as a possible adjuvant therapy capable of increasing the in vitro antitumor properties of Rituximab via apoptosis. However, further research is required to determine the in vivo antitumor effects of Nisin treatment.

\section{Acknowledgment}

We would like to thank all members of the Medical Biology Research Center for their discussion and support. This work was supported by Vice Chancellor for Research and Technology, Kermanshah University of Medical Sciences (97770).

The authors declare no conflict of interest.

6. Golay J, Zaffaroni L, Vaccari T, Lazzari M, Borleri GM, Bernasconi S, et al. Biologic response of B lymphoma cells to anti-CD20 monoclonal antibody Rituximab in vitro: CD55 and CD59 regulate complement-mediated cell lysis. Blood. 2000;95(12):3900-8.

7. Jazirehi AR, Gan X-H, De Vos S, Emmanouilides C, Bonavida B. Rituximab (antiCD20) selectively modifies Bcl-xL and apoptosis protease activating factor-1 (Apaf-1) expression and sensitizes human non-Hodgkin's lymphoma B cell lines to paclitaxel-induced apoptosis. Mol Cancer Ther. 2003;2(11):1183-93.

8. Goudarzi F, Asadi A, Afsharpour M, Jamadi RH. In Vitro Characterization and Evaluation of the Cytotoxicity Effects of Nisin and Nisin-Loaded PLA-PEG-PLA Nanoparticles on Gastrointestinal (AGS and KYSE-30), Hepatic (HepG2) and Blood (K562) Cancer Cell Lines. AAPS PharmSciTech. 2018;19(4):1554-1566. 
9. Lewies A, Wentzel JF, Miller HC, Du Plessis LH. The antimicrobial peptide Nisin $\mathrm{Z}$ induces selective toxicity and apoptotic cell death in cultured melanoma cells. Biochimie. 2018;144:28-40.

10. Ahmadi S, Ghollasi M, Hosseini HM. The apoptotic impact of Nisin as a potent bacteriocin on the colon cancer cells. Microb Pathog. 2017;111:193-197.

11. Kamarajan P, Hayami T, Matte B, Liu Y, Danciu T, Ramamoorthy A, et al. Nisin ZP, a Bacteriocin and Food Preservative, Inhibits Head and Neck Cancer Tumorigenesis and Prolongs Survival. PLoS One. 2015;10(7):e0131008.

12. Michel-Briand Y, Baysse C. The pyocins of Pseudomonas aeruginosa. Biochimie. 2002;84(56):499-510.

13. Paiva $A D$, de Oliveira MD, de Paula SO, Baracat-Pereira MC, Breukink E, Mantovani HC. Toxicity of bovicin HC5 against mammalian cell lines and the role of cholesterol in bacteriocin activity. Microbiology. 2012;158(Pt 11):2851-2858.

14. Cruz-Chamorro L, Puertollano MA, Puertollano E, de Cienfuegos GA, de Pablo MA. In vitro biological activities of magainin alone or in combination with
Nisin. Peptides. 2006;27(6):1201-9.

15. Gaspar D, Veiga AS, Castanho MARB. From antimicrobial to anticancer peptides. Front Microbiol. 2013;4:294.

16. Brotz H, Sahl HG. New insights into the mechanism of action of lantibiotics--diverse biological effects by binding to the same molecular target. J Antimicrob Chemother. 2000;46(1):1-6.

17. Joo NE, Ritchie K, Kamarajan P, Miao D, Kapila YL. Nisin, an apoptogenic bacteriocin and food preservative, attenuates HNSCC tumorigenesis via CHAC1. Cancer Med. 2012;1(3):295-305.

18. Moll GN, Clark J, Chan WC, Bycroft BW, Roberts GC, Konings WN, et al. Role of transmembrane $\mathrm{pH}$ gradient and membrane binding in Nisin pore formation. $\mathrm{J}$ Bacteriol. 1997;179(1):135-140.

19. Zainodini N, Hassanshahi G, Hajizadeh M, Khanamani Falahati-Pour S, Mahmoodi M, Mirzaei MR. Nisin Induces Cytotoxicity and Apoptosis in Human Asterocytoma Cell Line (SW1088). Asian Pac J Cancer Prev. 2018;19(8):2217-2222. 